\title{
Screening Policies and Prevention to Optimize Women's Health
}

\section{Elkady A*}

Consultant OBGYN, Police Force Hospital, Egypt

\section{Mini Review}

Volume 2 Special Issue 2

Received Date: May 15, 2017

Published Date: June 07, 2017

*Corresponding author: Adel Elkady, DGO - FICS - FRCOG, Consultant OBGYN, Police

Force Hospital, Cairo, Egypt, Email: dr@thewomenhealth.com

\section{Abstract}

Screening in medicine is the utilization of different tests and diagnostic modalities, aiming to diagnose or predict illnesses before them actually happen or are still in the asymptomatic stage. Once an individual shows a possible occurrence of a disease, preventive and treatment options can be offered to prevent disease occurrence or minimise the risks and complications of the disease. For an effective screening, the screening programs must comply with the WHO criteria for a good effective screening policy. Screening for cervical cancer, hepatitis B and HIV viral infections are few of too many examples of how may screening help to improve and optimize women's health.

The different types of screening policies include universal screening, selective high-risk population screening, opportunistic screening, and multimodal screening. All screening programmes must be aware of the possible limitations and disadvantages of any screening programme.

Screening may improve or save lives.

\section{Introduction and discussion}

Screening in medicine is methods and tests to identify healthy individuals who are at a higher risk of developing a disease later on. Screening used in a population to identify the detection of a possible presence of an as-yetundiagnosed disease in individuals who yet have no signs or symptoms of the disease. This can include individuals with pre-symptomatic or unrecognized symptomatic signs and symptoms. The intention of screening is to diagnose diseases earlier than it would be discovered. Without screening the disease may be discovered late, when symptoms appear or when it is too late to offer prevention or treatment. Screening presents the opportunity to offer prevention and treatment.Once screening identifies an individual at risk of developing a disease, management can be carried out to prevent disease occurrence or minimize the risks of this disease.

\section{The WHO Criteria for a Screening Test}

Screening must comply with the WHO criteria for a good screening test:

1. The condition sought should be an important health problem for the individual and community.

2. There should be an accepted treatment or useful intervention for patients with the disease.

3. The natural history of the disease should be adequately understood.

4. There should be a latent or early symptomatic stage.

5. There should be a suitable and acceptable screening test or examination. 
6. Facilities for diagnosis and treatment should be available.

7. There should be an agreed policy on whom to treat as patients.

8. Treatment started at an early stage should be of more benefit than treatment started later.

9. The cost should be economically balanced in relation to possible expenditure on medical care as a whole.

\section{Example of Screening Programmes}

A full list of examples of screening to optimize women's health is outside the merits of this editorial mini review; however, some examples of screening to optimize women's health include:

- $\quad$ Screening for cervical cancer. It includes pap smears and human papilloma virus (HPV) screening to detect the possibility of a woman developing cancer of the cervix at a later stage. Screening for cervical cancer is effective in both preventing cancer development and more importantly, preventing death from cervical cancer [1].

- Mammography. Mammography screening can produce up to $62 \%$ reduction in the breast cancer mortality [2].

- Diabetes or prediabetes. The WHO Diabetes Prevention Programme (DPP) concluded that screening and preventive strategies for type 2 diabetes iscost-effective. The lowest value for cost per quality of life year (QALY) gained) was USD 8,100/ person. They also concluded that there is direct evidence that the incidence of diabetes can be reduced in people at high risk of the future development of type 2 diabetes who may be identified as a result of activities directed towards diabetes detection [3].

- Screening for sexually transmitted infections. Sexually transmitted infections are a serious health hazard with current and long-term consequences. Prevention and treatment strategies can considerably reduce the health burden and are cost effective [4].

\section{Types of Screening}

- Universal mass screening: screening of a whole population or a subgroup of population. It is offered to all the population, irrespective of the risk status of the individual e.g. cervical screening of all females or HIV screening in all pregnant women.
- Selective or high-risk screening: it is conducted among high-risk populations only, e.g. screening highrisk pregnant women for gestational and established diabetes or those who have a higher risk of carrying a gene related (African populations have a relatively high prevalence of sickle cell anaemia)

- Multiphasic screening: Application of routine use of multiple tests to a large population at one time instead of carrying out separate screening tests for single diseases e.g. measuring blood pressure, urine analyses, ultrasound and biochemical screening of all pregnant women.

- Opportunistic screening: Either when an individual asks his health care provider for a check or a test to diagnose or predict a disease, or if a health care provider requests a test to check. Unlike an organised screening programme, opportunistic screening may not be checked or monitored.

\section{Limitations and Disadvantages of Screening}

- It involves the cost and use of medical resources when the majority of people screened do not need treatment.

- It may stretch an already exhausted medical services for cost and resources.

- It has adverse unfavourable effects of the screening procedures (stress and anxiety, discomfort, radiation and or chemical exposure).

- Stress and anxiety to the individual because it prolongs the time and knowledge waiting for an illness to happen or for cure.

- $\quad$ Stress, anxiety and extra cost of preventive measures because of over diagnosis (false positive) screening result (type I error).

- A false sense of security caused by under diagnosis or false negatives, with the resulting delay of final diagnosis (namely misdiagnosis with Type II error).

In summary, screening involves the employment of different tests to detect individuals who may develop a disease before it actually develops. It aims at offering preventive and treatment interventions to prevent disease occurrence, ameliorate its future health consequences or offer early treatment before it may be too late for any effective cure. Screening May save lives or improve the quality of life. 


\section{Conclusions}

In summary, screening involves the employment of different tests to detect individuals who may develop a disease before it actually develops. It aims at offering preventive and treatment interventions to prevent disease occurrence, ameliorate its future health consequences or offer early treatment before it may be too late for any effective cure.

Screening may save lives or improve the quality of life.

\section{References}

1. Landy R, Pesola F, Castañón A, Sasieni P (2016) Impact of cervical screening on cervical cancer mortality: estimation using stage-specific results from a nested case-control study. Br J Cancer 115(9): 11401146.
2. Morrell S, Taylor R, Roder D, Robson B, Gregory M, et al. (2017) Mammography service screening and breast cancer mortality in New Zealand: a National Cohort Study 1999-2011. Br J Cancer 116(6): 828839.

3. WHO Department of Non-communicable Disease Management Geneva (2003) Screening for Type 2 Diabetes Report of a World Health Organization and International Diabetes Federation meeting.

4. Kretzschmar M, Welte R, van den Hoek A, Postma MJ (2001) Comparative Model-based Analysis of Screening Programs for Chlamydia trachomatis Infections. Am J Epidemiol 153(1): 90-101. 